\title{
Box/Peanut Galaxies in the Near-IR
}

\author{
S. M. Baggett ${ }^{1}$ and J. W. MacKenty ${ }^{1}$ \\ STScI, 3700 San Martin Dr., Baltimore, MD 21218
}

\section{Introduction}

Galaxies with box/peanut-shaped (b/p) bulges have been known for some time (e.g., NGC 128 and NGC 7332 (Sandage 1961)). Observationally, b/p features are detected in edge-on systems, and are visible in images, contour maps, and brightness profiles as isophote sections that near minor axis are relatively flat and parallel to major axis (see Figure 1). Peanut-type bulges have isophotes indented at the intersection with the minor axis.

There have been several morphological surveys to date. The two most recent studies have found that about $20 \%$ of early-type galaxies and nearly $45 \%$ of all disk galaxies are b/p galaxies (see Shaw 1987, Dettmar 1989, and references therein) and that the $b / p$ galaxy properties appear similar to normal spirals in the optical, radio, and infrared. A few kinematical studies have been done: $\mathrm{b} / \mathrm{p}$ bulges are found to rotate more like disks than bulges (e.g., $150 \mathrm{~km} \mathrm{~s}^{-1}$ up to 6 kpc above the plane in NGC 128 (Jarvis 1990) and the velocities remain constant up to large $z$, implying cylindrical rotation (e.g., NGC 3079 has v constant out to $1.6 \mathrm{kpc}$ (Shaw et al. 1993)).

Several models have been suggested for $b / p$ galaxies; one of these is that $\mathrm{b} / \mathrm{p}$ galaxies may be barred galaxies seen edge-on: some models (Combes \& Sanders 1981, Combes, et al. 1990) exhibit a p-shape when the bar is viewed perpendicular to its long axis and a b-shape when viewed edge-on. Vertical resonances in the bar may supply the heating to generate the b/p shape (Friedli \& Pfenniger 1990, Pfenniger \& Friedli 1991). In these models, a bar viewed end-on appears round and the $b / p$ shape appears when the bar is viewed perpendicular to its long axis. A few b/p galaxies have been identified as possible edge-on SB galaxies, based on their surface brightness profiles (Hamabe \& Wakamatsu 1989; Dettmar \& Barteldrees 1990).

\section{Data and Analysis}

Optical CCD images were obtained at the KPNO $0.9 \mathrm{~m}$ telescope using the TEK 1 CCD $(512 \times 512)$ with 0.7 pixels and 6.5 total field of view. The galaxies observed were selected from lists of previously identified $\mathrm{b} / \mathrm{p}$ galaxies (Shaw 1987; DeSouza \& DosAnjos 1987). Near-IR images were obtained with the KPNO $1.3 \mathrm{~m}$, using SQIID (Simultaneous Quad-color Infrared Imaging Device)

\footnotetext{
${ }^{1}$ Visiting Astronomer, Kitt Peak National Observatory. KPNO is operated by AURA, Inc. under cooperative agreement with the National Science Foundation
} 

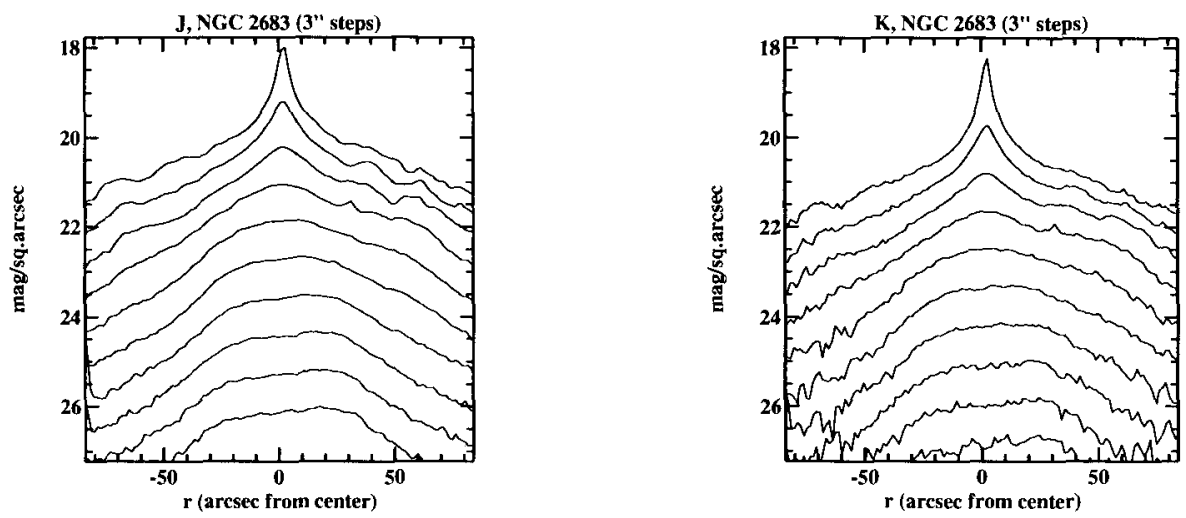

Figure 1. NGC $2683 J$ and $K$-band b/p cuts from southern side

camera equipped with PtSi detectors $(256 \times 256)$ with 1"4 pixels, total field of view $5^{\prime} .5$.

Six galaxies have been studied in detail so far; four of the six are $b / p$ while the other two appear to be normal edge-on galaxies. A striking result is that in the near-IR, each of the $b / p$ galaxies is seen to possess 2 well-defined spiral arms, which appear to start in the $b / p$ regions; the two non- $b / p$ galaxies show no such arms. Contour and profile plots confirm the visual inspection results. As an example, the b/p galaxy NGC 2683 surface brightness profiles parallel to the major axis at increasing $\mathrm{z}$ are shown in Figure 1 and the $K$-band contour plot is shown in Figure 2. A quantitative measure of the $b / p$ nature of the profiles parallel to the major axis was performed on all optical (Baggett 1993) and near-IR images (this study); these measurements confirm the results as well.

\section{Conclusions}

Results from recent SQIID $J H K$ observations of edge-on galaxies were presented. Six highly inclined galaxies from the literature $b / p$ lists have been examined in detail so far. Four galaxies exhibit obvious b/p features in the optical (Baggett 1993; Baggett \& Anderson, in prep.) and near-IR (this study) and two lack $b / p$ features.

As expected, the near-IR imaging provides unique information for edge-on galaxies, as the dust obscuration is greatly reduced. Four b/p galaxies show clear evidence of spiral arms emanating from the $b / p$ region; these arms are not visible in the optical images and don't appear in the non-b/p galaxy images.

The near-IR data is suggestive of a highly-inclined bar viewed end-on, with the spiral arms extending outward from the bar's end. Question for model builders: As a bar is viewed from edge-on to more face-on, at what inclination would the $b / p$ shape be expected to disappear? 

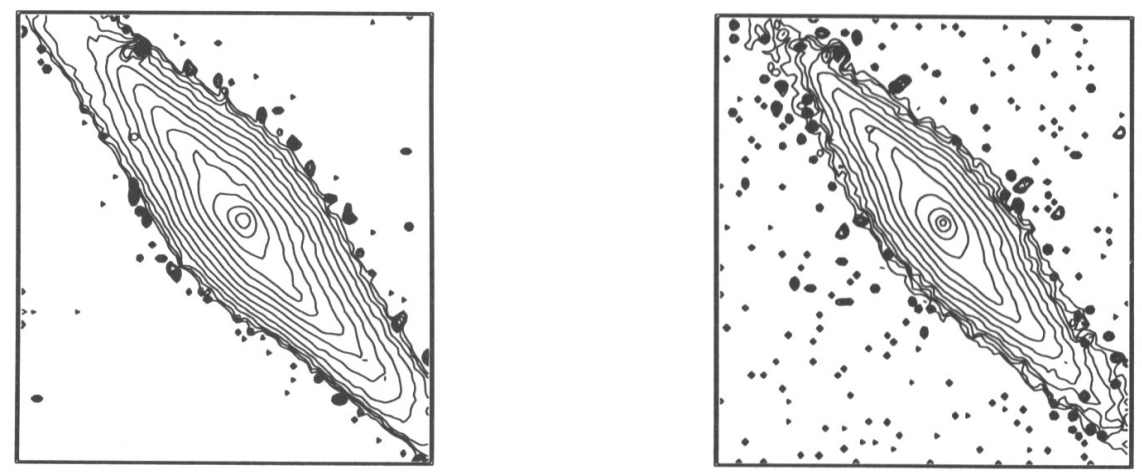

Figure 2. NGC 2683 $J$ and $K$-band image contours in 0.5 mag steps; images are 4.6 square

\section{References}

Baggett, S. M., Ph.D. Thesis 1993, New Mexico State University

Combes, F. \& Sanders, R. H. 1981, A\&A, 96, 164

Combes, F., Debbasch, F., Friedli, D., \& Pfenniger, D. 1990, A\&A, 233, 82

Dettmar, R.-J. 1989, in The World of Galaxies, H.C. Corwin \& L. Bottinelli, Berlin: Springer-Verlag, 229

Dettmar, R.-J. \& Barteldrees, A. 1990, in ESO Bulges of Galaxies Conference and Workshop Proceedings, No.35, 259

de Souza, R.E. \& dos Anjos, S. 1987, A\&AS, 70, 465

Hamabe, M. \& Wakamatsu, K. 1989, ApJ, 339, 783

Jarvis, B. 1990, in Dynamics and Interactions of Galaxies, Roland Wielen, Berlin: Springer-Verlag, 416

Pfenniger, D. \& Friedli, D. 1991, A\&A, 252, 75

Sandage, A. 1961, The Hubble Atlas of Galaxies, Washington, D. C.: Carnegie Institute of Washington, Publication 618

Shaw, M. A. 1987, MNRAS, 229, 691

Shaw, M., Wilkinson, A., \& Carter, D. 1993, A\&A, 268, 511 